Gastroenterologe 2007 · 2:179-185

DOI 10.1007/s11377-007-0078-5

Online publiziert: 8. März 2007

(c) Springer Medizin Verlag 2007

Schwerpunktherausgeber M. Fried, Zürich
W. Zingg

Service de prévention et contrôle de l'infection, Hôpitaux universitaires de Genève

\section{Die virale Gastroenteritis}

ten Studien untersuchen nur die Fälle im Krankenhaus oder allenfalls in der Arztpraxis), ist es für bis zu $70 \%$ der Hospitalisationen verantwortlich (• Abb. 1, 2, 3). Die Kosten der Rotavirusgastroenteritis in den USA werden auf 250 Mio. US-\$ pro Jahr geschätzt [27].

Viren verursachen aber nicht nur sporadische Fälle, sondern auch unzählige Epidemien in Krankenhäusern, Heimen, Schulen, Hotels, Restaurants und auf Kreuzfahrtschiffen. In Kinderkliniken, Spielgruppen und Kinderhorten sind Rotaviren für die Mehrzahl der Fälle verantwortlich ([3]; • Abb.4). Alle anderen Fälle werden in der Mehrheit durch Noroviren verursacht. Die hohe Infektiosität und die genetische Variabilität dieses Erregers sind ideale Voraussetzungen immer wiederkehrender Epidemien.

Zwischen 2001 und 2003 wurden in der Schweiz 73 größere Norovirusepidemien registriert: $34 \%$ in Pflegeheimen und $25 \%$ in Akutkrankenhäusern. Für weitere $13 \%$ wurden kontaminierte Nahrungsmittel verantwortlich gemacht [10]. Die $\mathrm{Zu}-$ satzkosten für eine nosokomiale Norovirusinfektion in der Schweiz konnten mit 787,50 Sfr pro Fall beziffert werden [30]. Hauptgrund dafür waren Einnahmeverluste, weil aufgrund einer hohen Befallsrate unter den Mitarbeitern, Abteilungen reduziert werden mussten. Rotavirusepidemien betreffen v. a. Kleinkinder und Säuglinge, weniger erwachsene Betreuungspersonen, die in der Regel eine ausreichende Immunität gegen schwere Krankheitsverläufe besitzen. Die pro Kind verursachten Kosten zwischen $1070 €$ (England) und $2602 €$ (Österreich; [12]) sind durch längere Hospitalisationszeiten von 3-6 Tagen bedingt.

\section{Pathophysiologie der wässrigen Diarrhö}

Die akute virale Gastroenteritis ist eine Infektion des Dünndarms. Die Viren dringen in die Enterozyten ein und in der Folge kommt es mikroskopisch zu einer Verkürzung und Verplumpung der Mikrovilli. Auf der Seite der Basolateralmembran
Mit geschätzten 4-6 Mio. Todesfällen Jahr gehört die Gastroenteritis weltweit zu den führenden Todesursachen. In den USA werden rund 200.000 Kinder pro Jahr mit einer akuten Gastroenteritis hospitalisiert, in Australien sind es 22.00o.

Die Epidemiologie der Erreger ist altersabhängig. Im Säuglings- und Kleinkindesalter dominieren die Rotaviren, jedoch sind die Noroviren nicht so selten, wie gemeinhin angenommen. Im Erwachsenenalter ist das Verhältnis umgekehrt, allerdings nimmt im Alter die Häufigkeit der Rotaviren, bedingt durch die verminderte Immunitätslage, wieder etwas $\mathrm{zu}$ (- Abb. 1). Obwohl das Rotavirus nur etwa $10 \%$ aller Gastroenteritiden in den ersten 5 Lebensjahren verursacht (die meis-
Abb. 1 Epidemiologie der viralen, sporadischen Gastroenteritis im Kleinkindes- und Erwachsenenalter in Holland (\%; [9])

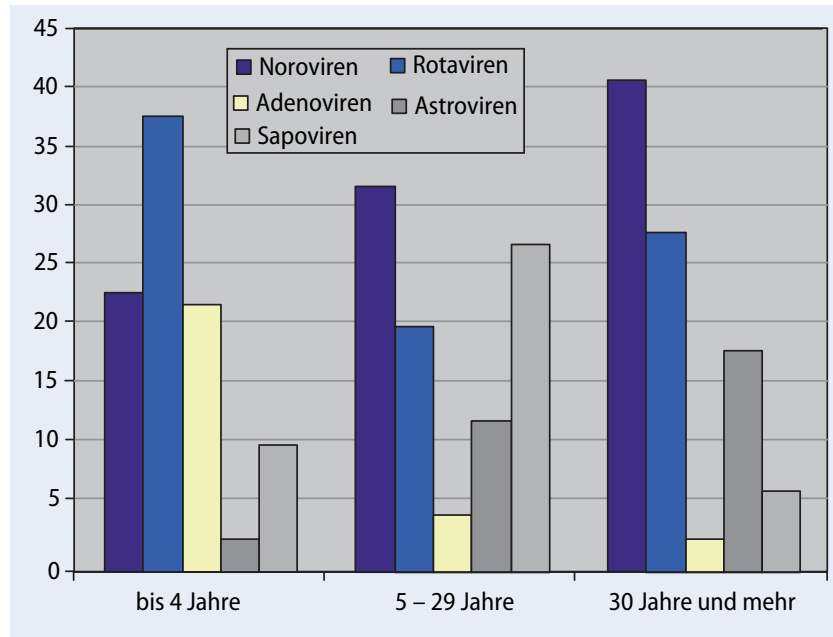




\section{Schwerpunkt: Akute gastrointestinale Infektionen}
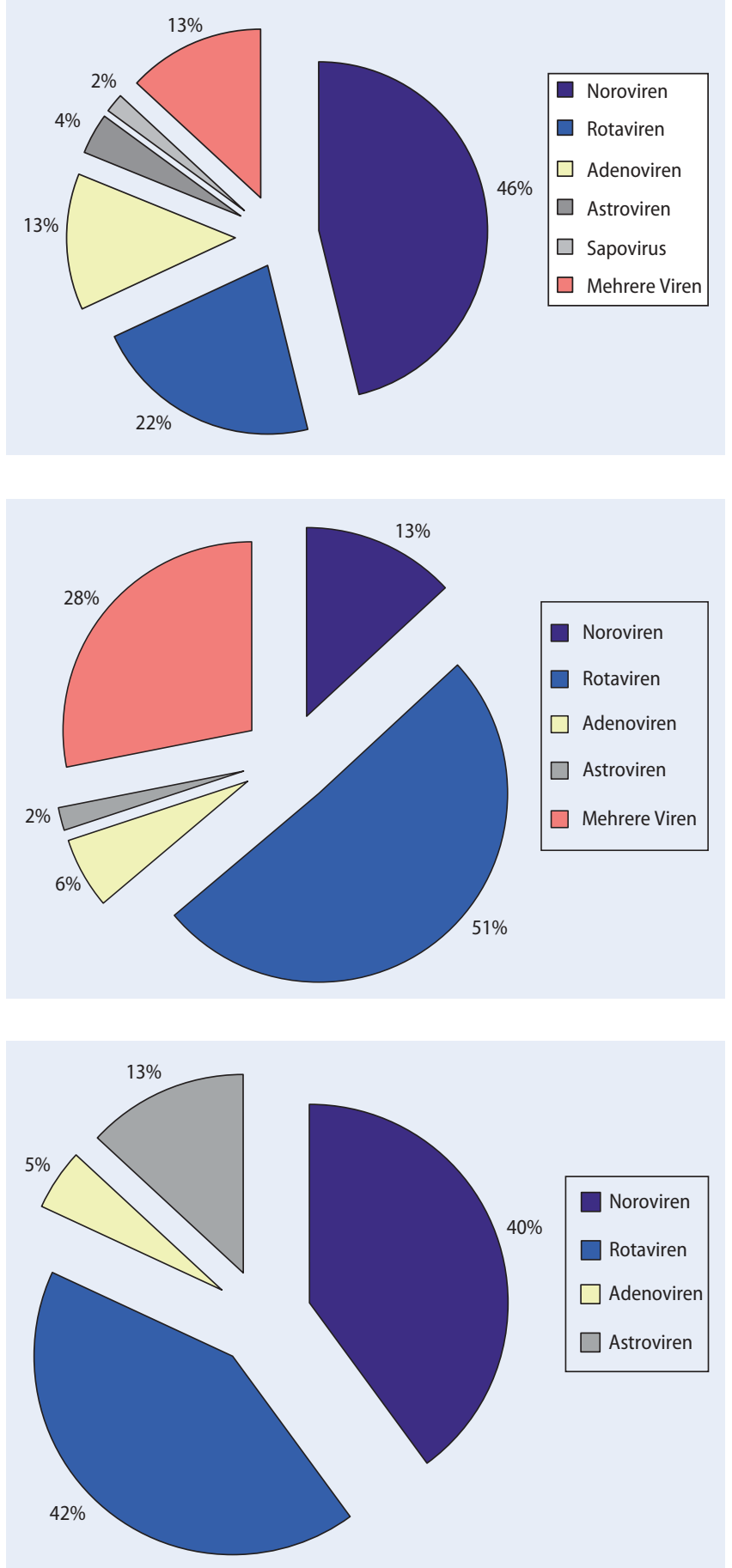

Abb. $2<$ Erreger sporadischer, viraler Gastroenteritiden bei Kindern $<5$ Jahren in England (East Anglia; [11])

Abb. $3<$ Erreger viraler Gastroenteritiden bei Kindern in einer Berliner Notaufnahme (29 Tage bis 15,5 Jahre; [22])

Abb. $4<$ Erreger viraler Gastroenteritiden bei 3-jährigen Kindern in einer Notaufnahme in Buenos Aires [6]

kommt es je nach Virus zu einer mehr oder weniger ausgeprägten mononukleären Infiltration. Die Mukosa wird in ihrer Architektur nicht verändert. Durch verminderte Enzymaktivität der Enterozyten werden Natrium und Glukose weniger gut resorbiert und es kommt zur Diarrhö. Die Aktivität der Adenylzyklase bleibt aber normal. Dies gewährleistet einen minimalen Nettotransport von Natrium durch die Zelle hindurch, die orale Rehydrierung mit natrium- und glukosehaltigen Lösungen bleibt daher effektiv. Nichtstrukturproteine gewisser Viren können als Enterotoxine wirken, die über einen Chloridefflux das Wasser im Darm binden und auf diese Weise zu einer Diarrhö führen.

\section{Erreger}

Viele Viren verursachen gastrointestinale Probleme. Nicht bei allen Viren ist die Gastroenteritis allerdings das vorherrschende Symptom. Noroviren, Rotaviren,
Adenoviren, Sapoviren und Astroviren sind für die überwiegende Mehrzahl der Fälle verantwortlich. Meist gehören sie zu Familien, deren andere Vertreter nicht humanpathogen sind, jedoch ein zur Gastroenteritis vergleichbares Krankheitsbild bei Tieren verursachen.

\section{Noroviren}

Noroviren, früher elektronenmikroskopisch als „small round structured viruses“ und später als „Norwalk-like-Viren“ bezeichnet, wurden zum ersten Mal 1972 isoliert. Anlass war eine Epidemie an einer Schule in Norwalk, Ohio. Die Krankheit wurde als „winter vomiting disease“ bezeichnet, entsprechend der Saisonalität des Virus. Mittlerweile gehört es zusammen mit dem Sapovirus, ehemals „Sapporo-like-Virus", und einem weiteren Vertreter zur Familie der Caliciviren. Noroviren sind Einzelstrang-RNA-Viren mit hoher genetischer Variabilität. Die induzierte Immunität ist nur von kurzer Dauer. Unterschieden werden 4 Genotypen: Die Gruppen I, II und IV sind humanpathogen. Die unbehüllten Viren sind mit einer Größe von 20-25 nm sehr klein.

Die Krankheitsdauer ist in der Regel kurz. Nach einer Inkubationszeit von 24$48 \mathrm{~h}$ beginnt die Krankheit mit Nausea und Erbrechen ohne Fieber, gefolgt von einer wässrigen Diarrhö für 1-2 Tage. Prolongierte Verläufe bis 3 Wochen sind beschrieben, hauptsächlich bei Patienten mit kompromittiertem Immunsystem. Noroviren können mittels PCR-Analyse im nativen Stuhl nachgewiesen werden [28]. Diese Untersuchung macht jedoch nur in der Epidemieabklärung Sinn. Sporadische Fälle akuter Gastroenteritis brauchen in der Regel keine Erregerdiagnostik.

Bereits 10 Viruspartikel genügen für eine Infektion [7]. Die Virusausscheidung im Stuhl beginnt kurz vor der Diarrhö, erreicht ihren Höhepunkt am 1. bis 2. Tag nach Krankheitsbeginn und dauert bis zu 14 Tage [24]. Die Abnahme der Ausscheidung ist aber nicht linear: Bereits 2 Tage nach Symptomende ist die Zahl der Viren im Stuhl sehr gering. Das Virus kann lichtgeschützt über Tage auf Oberflächen und in Flüssigkeiten überleben, besonders bei tieferen Temperaturen. Da es für eine Infektion nur wenige Viruspartikel 
braucht, sind Tische, Stühle, Spielsachen und Speisen perfekte Vektoren für die Virusübertragung.

\section{Bereits 10 Viruspartikel genügen für eine Infektion}

Die hohe genetische Variabilität des Virus verhindert eine lang anhaltende, robuste Immunität. Dennoch entwickelt nicht jeder nach Viruskontakt Symptome. Die Norovirusinfektion hängt mit dem FUT2-

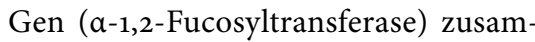
men. Es konnte gezeigt werden, dass Individuen, welche das Gen nicht exprimieren $\left(\mathrm{FUT}_{2}{ }^{-/-}\right)$, etwa $20 \%$ der europäischen Bevölkerung, eine hohe Resistenz gegenüber einer Norovirusinfektion aufweisen [17].

Patienten mit einer Norovirusgastroenteritis im Krankenhaus oder in einer Pflegeinstitution müssen isoliert werden. Die Pflege erfolgt mit Schürze, Handschuhen und Mundschutz. Im Falle einer Epidemie müssen zusätzliche Hygienemaßnahmen frühzeitig und großzügig eingeleitet werden [14]:

- Betroffene Angestellte müssen bis mindestens 2 Tage nach Symptomende beurlaubt werden,

- Patienten müssen konsequent isoliert oder kohortiert werden,

- Desinfektionsmaßnahmen müssen mit geeigneten Mitteln intensiviert werden.

Übliche Desinfektionsmittel sind gegen Noroviren nicht wirksam. Zur Händedesinfektion wird eine alkoholische Lösung mit 95\% Ethanol benötigt, für die Flächendesinfektion eine Natriumhypochloritlösung 5000 ppm (Javel). Glutaraldehyde und jodbasierte Desinfektionsmittel inaktivierten das Virus ebenfalls.

\section{Rotaviren}

Rotaviren sind unbehüllte $70 \mathrm{~nm}$ messende Viren mit doppelsträngiger RNA und gehören zur Familie der Reoviridae. Die äußeren Kapsidantigene $\mathrm{VP}_{4}$ und $\mathrm{VP}_{7}$ definieren 7 Genogruppen (A-G). Die humanen Gastroenteritiden werden meist durch Viren der Gruppe A verursacht, Fälle mit den Gruppen B und C sind jedoch beschrieben.
Nach einer Inkubationszeit von 2-4 Tagen beginnt die Krankheit mit Nausea, Erbrechen und Fieber. Etwa 1-2 Tage später entwickelt sich eine ausgeprägte, wässrige Diarrhö für 3-8 Tage. In 1,2-2,6\% der Fälle treten benigne, zerebrale Krampfanfälle auf, nicht zu verwechseln mit Fieberkrämpfen. Rotaviren können sowohl im Liquor als auch im Blut nachgewiesen werden $[8,20]$. Die Virusausscheidung im Stuhl beginnt einen Tag vor Ausbruch der Diarrhö und persistiert bis zu 3 Wochen. Bei Patienten mit kompromittiertem Immunsystem finden sich Viren noch nach mehreren Wochen im Stuhl. In der Akutphase werden bis $\mathrm{zu}^{1 \mathrm{O}^{12}}$ Mikroorganismen/g Stuhl ausgeschieden. Die Diagnose geschieht in der Regel mittels ELISA im Stuhl. PCR-Analysen sind allerdings sensitiver [29].

\section{- Die Rotavirusgastreoenteritis ist eine Erkrankung des Kindesalters.}

Im Alter von 2 Jahren weisen bereits 62\% der Kinder spezifische Antikörper auf. Die Infektion führt in der Regel zu einer guten und lange anhaltenden Immunität, die nicht grundsätzlich vor einer Neuinfektion schützt, jedoch vor einem schweren Verlauf. Bei Erwachsenen machen die Rotaviren deshalb nur 1-4\% der symptomatischen Gastroenteritiden aus, der Krankheitsverlauf ist milder. Betroffen sind zumeist Eltern kranker Kinder (40\%). Mit Abnahme der Immunität im Alter verursachen Rotaviren erneut schwere Gastroenteritiden und Epidemien in Alters- und Pflegeheimen.

Die Kontaktinfektion geschieht fäkaloral zwischen Personen und Oberflächen oder Gegenständen, wo das Virus bis zu 10 Tage nachgewiesen werden kann. Nur gerade 10 Viruspartikel braucht es für eine Infektion. Rotaviren können mit 70\% Ethanol, 2\% Glutaraldehyd, 70\% Isopropylalkohol oder Jodpräparaten desinfiziert werden [25]. Die krankenhaushygienischen Maßnahmen bei sporadischen und epidemischen Fällen sind mit denjenigen bei Noroviren vergleichbar, jedoch reichen die im Alltag üblicherweise verwendeten Desinfektionsmittel aus. Eine Epidemie im Krankenhaus oder im Kinderhort beschränkt sich meist auf die Kin-
Gastroenterologe 2007 · 2:179-185

DOI 10.1007/s11377-007-0078-5

(c) Springer Medizin Verlag 2007

\section{W. Zingg}

Die virale Gastroenteritis

\section{Zusammenfassung}

Viren sind die häufigste Ursache der akuten Gastroenteritis und nicht nur in den Entwicklungsländern ein Problem. Rotaviren bilden bei Säuglingen und Kleinkindern und Noroviren in allen Altersgruppen die Haupterreger. Neben sporadischen Fällen verursachen sie auch bei uns Epidemien in Krankenhäusern, Pflegeheimen, Schulen, Kinderkrippen, Hotels und Restaurants. Während die medizinische Betreuung sporadischer Fälle außer bei Säuglingen kaum Probleme bietet, ist das Management bei Epidemien aufwändig und zeitraubend. Der vorliegende Beitrag ist eine Übersicht über die wichtigsten Erreger, deren Pathogenese, Therapie und die krankenhaushygienische Betreuung.

Schlüsselwörter

Gastroenteritis · Norovirus · Rotavirus . Probiotika

\section{Viral gastroenteritis}

\section{Abstract}

The acute gastroenteritis is a problem not only in developing countries but also in Europe. Rotaviruses cause severe disease in the young infant and noroviruses cause milder disease in all age groups. They are responsible for countless outbreaks in hospitals, nurseries, day care centers, hotels and restaurants. Rehydration of infants and neonates can be challenging, but handling of viral gastroenteritis in general is easy. Outbreak management on the other hand can be laborious and time consuming. This report is an overview on pathogenesis, management and epidemiology of the most important viruses causing acute gastroenteritis.

\section{Keywords}

Gastroenteritis · Norovirus · Rotavirus .

Probiotics 


\section{Schwerpunkt: Akute gastrointestinale Infektionen}

Tab. 1 Klinische Beurteilung des Dehydratationsgrads von Säuglingen und

Kleinkindern

\begin{tabular}{|c|c|c|c|}
\hline \multirow[t]{2}{*}{ Symptome } & \multicolumn{3}{|l|}{ Dehydratation } \\
\hline & Leicht & Mittel & Schwer \\
\hline Allgemeinzustand & Normal & Müde, irritabel & $\begin{array}{l}\text { Lethargisch, schlapp, } \\
\text { apathisch }\end{array}$ \\
\hline Augen & Normal & Etwas eingesunken & Haloniert \\
\hline Tränen & Normal & Vermindert & Abwesend \\
\hline Mundschleimhaut & Feucht & Trocken & Ausgetrocknet \\
\hline Hautfalte & Normal & $<2 \mathrm{~s}$ & $>2 \mathrm{~s}$ \\
\hline Trinkverhalten & $\begin{array}{l}\text { Normal, evtl. etwas } \\
\text { vermindert }\end{array}$ & $\begin{array}{l}\text { Durstig, begieriges } \\
\text { Trinken }\end{array}$ & Kein Trinken \\
\hline Urinausscheidung & Normal & Vermindert & Keine Ausscheidung \\
\hline $\begin{array}{l}\text { Gewichtsverlust } \\
\text { (\% des Körpergewichts) }\end{array}$ & $<5$ & $5-10$ & $>10$ \\
\hline Puls & Normal & Erhöht & Tachykardie \\
\hline Atmung & Normal & Schnell & Tief \\
\hline
\end{tabular}

der. Betreuungspersonen erkranken eher sporadisch.

\section{Seltenere Erreger}

\section{Adenoviren}

Adenoviren verursachen verschiedene Krankheitsbilder. Mindestens 51 Serotypen sind bisher beschrieben. Die meisten Gastroenteritiden werden durch die enterischen Typen 40 und 41 verursacht, gefolgt von den Serotypen 3, 7 und 31. Mit 4 Jahren weisen 50\% der Kinder Antikörper gegen die enterischen Typen 40 und 41 auf.

Nach einer Inkubationsphase von 810 Tagen beginnt die Krankheit mit Fieber und Erbrechen, gefolgt von einer wässrigen Diarrhö für weitere 7 Tage. Gelegentlich bestehen gleichzeitig auch Symptome der oberen Atemwege. Die Übertragung erfolgt fäkal-oral, in der Regel von Person zu Person und weniger über Oberflächen. Die Viren induzieren eine gute, lebenslange Immunität. Deshalb ist die Adenovirusgastroenteritis hauptsächlich eine Erkrankung des Kindesalters und für 5-20\% der Hospitalisationen in dieser Altersgruppe verantwortlich. HIV-positive Patienten entwickeln gelegentlich eine Adenoviruskolitits. Die Diagnose erfolgt in der Regel mittels ELISA im Stuhl. Alternativ dazu gibt es auch eine PCR-Analyse. Die Virusausscheidung beschränkt sich in der Regel auf die Dauer der Diarrhö, asymptomatische Ausscheider wurden allerdings beschrieben [16].

\section{Astroviren}

Die Astroviren sind kleine, 28-35 nm messende, unbehüllte Viren mit einer Einzelstrang-RNA. Im Elektronenmikroskop imponieren sie als 5- bis 6-zackige "Sterne“. 2 Gruppen werden unterschieden, wobei die humanpathogenen Serotypen 1, 3, 4, 5 und 8 der Mamastroviren Erkrankungen beim Menschen verursachen [13].

Nach einer Inkubationszeit von 24-36 h beginnt die Krankheit mit Nausea, Erbrechen und Fieber, gefolgt von einer Diarrhö über 1-4 Tage. Im Vergleich zu anderen viralen Erregern ist der Verlauf milder, auBer beim Serotyp 3, der durchaus schwere Verläufe verursachen kann. Im Stuhl werden die Viren bis zu 2 Wochen ausgeschietiven Stuhl nachgewiesen werden. Auch die Astrovirusgastroenteritis ist eine Erkrankung des Kindesalters. Im Alter von 10 Jahren finden sich bereits bei $75 \%$ der Kinder spezifische Antikörper.

\section{Enteroviren}

Coxsackie-, Echo- und andere Enteroviren gehören zu den Picornaviridae. Sie verursachen nur selten eine akute Gastroenteritis im klassischen Sinn. Meist handelt es sich um eine Allgemeinerkrankung mit einer Diarrhö bei Symptombeginn, bevor andere Organsysteme in den Vordergrund treten. Die Echoviren 6, 7, 11, 18, 25 und 30 und die Coxsackieviren A9, $\mathrm{B}_{2}, \mathrm{~B}_{3}$ und $\mathrm{B}_{4}$ können sporadische Gastroenteritiden verursachen, insbesondeden. Virus-RNA kann mittels PCR im na- re bei Säuglingen in den ersten Lebensmonaten.

\section{Parechoviren}

Humane Parechoviren gehören, wie die Enteroviren, zu den Picornaviridae. Früher wurden die gastroenteritisassoziierten Typen den Enteroviren zugeordnet und als Enterovirus 22 und 23 bezeichnet. Aktuell werden 4 Serotypen (HPeV1-4) unterschieden [4]. Bisher wurden Parechoviren nur bei Kindern unter 3 Jahren gefunden. $\mathrm{HPeV} 1$ und $\mathrm{HPeV}_{2}$ verursachen Infektionen der oberen Atemwege mit gastrointestinalen Symptomen. $\mathrm{HPeV}_{3}$ finden sich v. a. bei Neugeborenen und kleinen Säuglingen mit sepsisähnlichem Krankheitsbild und ZNS-Beteiligung [5].

\section{Coronaviren}

Die Viren HCoV-OC43 und $229 \mathrm{E}$ sind für etwa ein Drittel aller Schnupfenfälle verantwortlich. Sie verursachen gelegentlich aber auch gastrointestinale Symptome mit Diarrhö, insbesondere bei HIV-positiven Patienten [26]. Dies trifft auch für das SARS-CoV zu, der wohl bekannteste Vertreter der Coronaviren. Bis zu 40\% der Patienten entwickelten gastrointestinale Symptome, teilweise als alleinige klinische Präsentation [23].

\section{Toroviren}

Toroviren gehören zur Familie der Coronaviridae. Sie sind behüllt, etwa 10o$140 \mathrm{~nm}$ groß und besitzen eine Einzelstrang-RNA. Die humanpathogenen Viren verursachen akute und chronische Durchfallerkrankungen bei Kindern, teilweise in Assoziation mit enteroaggregativen E. coli [15]. Kürzlich wurden Toroviren auch im Stuhl von Kindern mit nekrotisierender Enterokolitis gefunden [19]. Der Zusammenhang ist jedoch noch nicht klar.

\section{Picobirnaviren}

Picobirnaviren sind unbehüllte, $35-41 \mathrm{~nm}$ große RNA-Viren. Seit 1988 wird das Virus mit sporadischen akuten Durchfallerkrankungen in Verbindung gebracht, insbesondere bei immunsupprimierten Patienten. Kürzlich fanden sich Picobirnaviren in Stuhlproben einer durch Caliciviren verursachten Epidemie [2]. 
Hier steht eine Anzeige.

黛 Springer 


\section{Aichivirus}

Das Aichivirus ist ein RNA-Virus und gehört zu den Picornaviren. Es ist besonders in Südostasien endemisch und wurde, auBer im Zusammenhang mit einer durch Austern induzierten Epidemie, vor allem bei Reiserückkehrern aus diesen Gebieten beschrieben. Letztes Jahr wurde über erste Fälle in Brasilien und Deutschland berichtet [21].

\section{Therapie}

Die Therapie der viralen Gastroenteritis ist rein symptomatisch. Im Prinzip geht es lediglich darum, das Flüssigkeitsdefizit und mögliche Elektrolytverschiebungen auszugleichen. Die Flüssigkeitssubstitution soll möglichst ambulant und mit einer Elektrolyt-Glukose-Lösung erfolgen, sowohl bei Kindern als auch bei Erwachsenen. Bei Kindern richtet sich die Art der Substitution nach dem Dehydratations$\operatorname{grad}(\bullet$ Tab. 1)

$\mathrm{Zu}$ Beginn der Gastroenteritis, wenn Nausea und Erbrechen im Vordergrund stehen, ist es schwierig, Kindern die nötige Flüssigkeit oral zu verabreichen. Meist besteht zu diesem Zeitpunkt aber noch keine Dehydratation und die Rehydrierung kann langsam mit einem Kaffeelöffel erfolgen. Zwischen den einzelnen Gaben muss eine Pause von etwa 1 min eingelegt werden. Das Prozedere wird so lange fortgeführt, bis das Kind nicht mehr durstig ist. Auf diese Weise kann eine mittelschwere bis schwere Dehydratation vermieden werden.

Die parenterale Rehydrierung ist nur bei einer mittleren bis schweren Dehydratation indiziert. Hierfür sollen natriumhaltige Präparate (o,9\%- $\mathrm{NaCl}$ oder Ringer-Glukose) verwendet werden, um eine Hyponatriämie (Krämpfe, Exitus) zu vermeiden. Bei allzu großzügiger Volumensubstitution kommt es zu Lidödemen. Ein Kind mit mittelschwerer Dehydratation kann ambulant auch mit Volumenboli von je $20 \mathrm{ml} / \mathrm{kgKG}$ behandelt werden, bis es in der Lage ist, die weitere Flüssigkeit oral zu sich zu nehmen. Falls eine Trinkschwäche besteht oder die Betreuung des Kindes zu Hause nicht gewährleistet ist, muss es hospitalisiert werden. Ein Kind mit schwerer Dehydratation muss auf jeden Fall hospitalisiert werden.
Rotaviren können eine temporäre Laktose-Intoleranz verursachen. Diese ist reversibel, die Umstellung auf eine laktosefreie Nahrung ist nicht indiziert. Gestillte Kinder sollen weiter gestillt werden.

Die auch bei uns zugelassenen Probiotika Saccharomyces boulardii (Perenterol $^{\oplus}$, Florosan ${ }^{\circledast}$, Ultra Levure ${ }^{\circledast}$ ), Lactobacillus acidophilus (Lactoferment ${ }^{\oplus}$ /10, Lacté$\mathrm{ol}^{\oplus}$ 5, Infloran ${ }^{\circledR}$ ) und Bifidobacterium lactis/infantis (Infloran ${ }^{\circledR}$ ) senken die Dauer der Diarrhö bei akuten viralen, nicht aber bei bakteriellen Gastroenteritiden [1].

\section{Prävention}

Im Februar 2006 wurde in den USA ein pentavalenter, boviner Impfstoff zur Impfung gegen Rotaviren zugelassen, nachdem ein 1998 eingeführter Impfstoff wieder vom Markt genommen werden musste, weil eine erhöhte Inzidenz von Invaginationen bei geimpften Kindern festgestellt wurde. Der neue Impfstoff verhindert eine Gastroenteritis zu $74 \%$ und schwere Formen gar zu 98\%.

Eine Impfung gegen Noroviren ist noch nicht erhältlich. Weil das Norovirus aber nicht kultiviert werden kann, wird es wohl noch einige Zeit dauern, bis ein Impfstoff zur Verfügung steht. Immerhin konnte man zeigen, dass multivalente Antigene zu einer Immunantwort mit messbaren Antikörpern auf der Mukosa führen [18].

Ein Nutzen der regelmäßigen Einnahme von Probiotika zur Prävention viraler Gastroenteritiden konnte nicht gezeigt werden.

\section{Fazit für die Praxis}

Die virale Gastroenteritis ist ein alltägliches Problem der Allgemeinärzte, Kinderärzte und Notfallstationen. Bei entsprechender Verdachtsdiagnose kann im ambulanten Fall auf Laboruntersuchungen weitgehend verzichtet werden. Im Falle einer Epidemie im Krankenhaus oder in einer Pflegeinstitution lohnt sich allerdings die genaue Differenzierung des Keims. Krankenhaushygienische Maßnahmen müssen dann dem Erreger angepasst, frühzeitig und konsequent umgesetzt werden. Kompromisse lohnen sich in der Regel nicht. Die Therapie der sporadischen Gastroenteritis kann in den meis- ten Fällen ambulant durchgeführt werden, auch bei Säuglingen und Kleinkindern. Die orale Rehydratation ist der Infusion vorzuziehen, weil sie einfacher und schonender ist und weniger Komplikationen verursacht. Selbst Säuglinge mit ausgeprägter Trinkschwäche, die stationär behandelt werden müssen, können mittels Magensonde schonend mit oralen Rehydratationslösungen rehydriert werden.

\section{Korrespondierender Autor}

\section{Dr.W.Zingg}

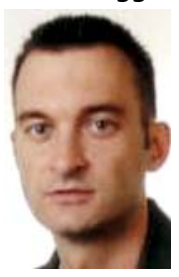

Service de prévention et contrôle de l'infection, Hôpitaux universitaires de Genève,

24 rue Micheli-du-Crest, 1211 Genève 14 walter.zingg@hcuge.ch

Interessenkonflikt. Es besteht kein Interessenkonflikt. Der korrespondierende Autor versichert, dass keine Verbindungen mit einer Firma, deren Produkt in dem Artikel genannt ist, oder einer Firma, die ein Konkurrenzprodukt vertreibt, bestehen. Die Präsentation des Themas ist unabhängig und die Darstellung der Inhalte produktneutral.

\section{Literatur}

1. Allen SJ, Okoko B, Martinez E et al. (2004) Probiotics for treating infectious diarrhoea. Cochrane Database Syst Rev 2: CD003048

2. Banyai K, Jakab F, Reuter G et al. (2003) Sequence heterogeneity among human picobirnaviruses detected in a gastroenteritis outbreak. Arch Virol 148: 2281-2291

3. Bartlett AV, Moore M, Gary GW (1985) Diarrheal illness among infants and toddlers in day care centers. I. Epidemiology and pathogens. J Pediatr 107: 495-502

4. Benschop KS, Schinkel J, Minnaar RP et al. (2006) Fourth human parechovirus serotype. Emerg Infect Dis 12: 1572-1575

5. Benschop KS, Schinkel J, Minnaar RP et al. (2006) Human parechovirus infections in Dutch children and the association between serotype and disease severity. Clin Infect Dis 42: 204-210

6. Bereciartu A, Bok K, Gomez J et al. (2002) Identification of viral agents causing gastroenteritis among children in Buenos Aires, Argentina. J Clin Virol 25: 197-203

7. Berg DE, Kohn MA, Farley TA et al. (2000) Multi-state outbreaks of acute gastroenteritis traced to fecal-contaminated oysters harvested in Louisiana. J Infect Dis 181: S381-S386

8. Blutt SE, Kirkwood CD, Parreno V et al. (2003) Rotavirus antigenaemia and viraemia: a common event? Lancet 362: 1445-1449

9. De Wit MA, Koopmans MP, Kortbeek LM et al. (2001) Etiology of gastroenteritis in sentinel general practices in the Netherlands. Clin Infect Dis 33: 280-288 
10. Fretz R, Svoboda P, Luthi TM et al. (2005) Outbreaks of gastroenteritis due to infections with norovirus in Switzerland, 2001-2003. Epidemiol Infect 133: 429-437

11. Froggatt PC, Barry Vipond I, Ashley CR et al. (2004) Surveillance of norovirus infection in a study of sporadic childhood gastroenteritis in South West England and South Wales, during one winter season (1999-2000). J Med Virol 72: 307-311

12. Gleizes OO, Desselberger U, Tatochenko V et al. (2006) Nosocomial rotavirus infection in European countries: a review of the epidemiology, severity and economic burden of hospital-acquired rotavirus disease. Pediatr Infect Dis J 25: S12-S21

13. Jakab F, Walter JE, Berke T et al. (2003) Molecular characterization and sequence analysis of human astroviruses circulating in Hungary. FEMS Immunol Med Microbiol 39: 97-102

14. Khanna N, Goldenberger D, Graber P et al. (2003) Gastroenteritis outbreak with norovirus in a Swiss university hospital with a newly identified virus strain. J Hosp Infect 55: 131-136

15. Koopmans M, Goosen ES, Lima AA et al. (1997) Association of torovirus with acute and persistent diarrhea in children. Pediatr Infect Dis J 16: 504-507

16. Kotloff KL, Losonsky GA, Morris JG et al. (1989) Enteric adenovirus infection and childhood diarrhea: an epidemiologic study in three clinical settings. Pediatrics 84: 219-225

17. Le Pendu J, Ruvoen-Clouet N, Kindeberg E et al. (2006) Mendelian resistance to human norovirus infections. Semin Immunol 18: 375-386

18. LoBue AD, Lindesmith L, Yount B et al. (2006) Multivalent norovirus vaccines induce strong mucosal and systemic blocking antibodies against multiple strains. Vaccine 24: 5220-5234

19. Lodha A, Silva N de, Petric M et al. (2005) Human torovirus: a new virus associated with neonatal necrotizing enterocolitis. Acta Paediatr 94: 1085-1088

20. Lynch M, Lee B, Azimi P et al. (2001) Rotavirus and central nervous system symptoms: cause or contaminant? Case reports and review. Clin Infect Dis 33: 932-938

21. Oh DY, Silva PA, Hauroeder B et al. (2006) Molecular characterization of the first Aichi viruses isolated in Europe and in South America. Arch Virol 151: 1199-1212

22. Oh DY, Gaedicke G, Schreier E et al. (2003) Viral agents of acute gastroenteritis in German children: prevalence and molecular diversity. J Med Virol 71: 82-93

23. Peiris JS, Chu CM, Cheng VC et al. (2003) Clinical progression and viral load in a community outbreak of coronavirus-associated SARS pneumonia: a prospective study. Lancet 2003 361: 1767-1772

24. Rockx B, De Wit M, Vennema H et al. (2002) Natural history of human calicivirus infection: a prospective cohort study. Clin Infect Dis 35: 246-253

25. Sattar SA, Rapahel RA, Lochnan H et al. (1983) Rotavirus inactivation by chemical disinfectants and antiseptics used in hospitals. Can J Microbiol 29: 1464-1469

26. Schmidt W, Schneider T, Heise W et al. (1996) Stool viruses, co-infections, and diarrhoea in HIV-infected patients. J Acquire Immune Def Syndr Hum Retrovirol 13: 33-39

27. Tucker AW, Haddix AC, Bresee JS et al. (1998) Costeffectiveness analysis of a rotavirus immunization program for the United States. JAMA 279: 13711376

28. Vinje J, Vennema H, Maunula L et al. (2003) International collaborative study to compare reverse transcriptase PCR assays for detection and genotyping of noroviruses. J Clin Microbiol. 41: 1423-1433
29. Wilde J, Yolken R, Willoughby et al. (1991) Improved detection of rotavirus shedding by polymerase chain reaction. Lancet 337: 323-326

30. Zingg W, Colombo C, Jucker T et al. (2005) Impact of an outbreak of norovirus infection on hospital resources. Infect Control Hosp Epidemiol 26: 263-267

\section{Parallele Evolution von Mensch und Helicobacter pylori}

Mehr als die Hälfte der Weltbevölkerung ist mit dem gramnegativen Bakterium Helicobacter pylori infiziert, das die Magenschleimhaut besiedelt und als Erreger verschiedener Magenerkrankungen identifiziert wurde. Forscher aus Berlin, Cambridge und Hannover haben die Koevolution von Mensch und Bakterium untersucht und herausgefunden, dass Helicobacter mindestens seit Beginn der letzten globalen Ausbreitung der Menschheit vor 60.000 Jahren mit dem Menschen assoziiert ist [B. Linz, F. Balloux, Y. Moodley et al (2007) An African origin for the intimate association between humans and Helicobacter pylor. Nature 445, 915-918].

Sechs genetisch verschiedene Haupttypen des Bakteriums lassen sich unterscheiden: drei afrikanische und zwei asiatische Typen sowie ein europäischer Typ. Ein Vergleich repräsentativer DNA-Sequenzen aus dem Bakteriengenom mit menschlicher DNA zeigte, dass sich das Erbgut der Bakterien zu dem des menschlichen „Trägers" parallel verändert hat, so dass sich populations $\neg$ geschichtliche Ereignisse der Menschheitsgeschichte - beispielsweise Völkerwanderungen - im bakteriellen Genom abbilden.

Quelle:

Medizinische Hochschule Hannover 